\title{
The association between negative attention biases and symptoms of depression in a community sample of adolescents
}

Belinda Platt, Susannah E Murphy, Jennifer Y.F. Lau

Adolescence is a vulnerable time for the onset of depression. Recent evidence from adult studies suggests not only that negative attention biases are correlated with symptoms of depression, but that reducing negative attention biases through training can in turn reduce symptomology. The role and plasticity of attention biases in adolescent depression, however, remains unclear. This study examines the association between symptoms of depression and attention biases, and whether such biases are modifiable, in a community sample of adolescents. We report data from 105 adolescents aged 13-17 who completed a dot-probe measure of attention bias before and after a single session of visual searchbased cognitive bias modification training. This is the first study to find a significant association between negative attention biases and increased symptoms of depression in a community sample of adolescents. Contrary to expectations, we were unable to manipulate attention biases using a previously successful cognitive bias modification task. There were no effects of the training on positive affect and modest effects of the training on negative affect which were evident for participants with more symptoms of depression are interpreted with caution. Our data replicate those from the adult literature, which suggest that adolescent depression is a disorder associated with negative attention biases, although we were unable to modify attention biases in our study. We identify numerous parameters of our methodology which may explain these null training effects, and which could be addressed in future cognitive bias modification studies of adolescent depression. 
1 Title: The association between negative attention biases and symptoms of depression in a

2 community sample of adolescents

3

4 Authors: Belinda Platt ${ }^{1,2}$, Susannah E. Murphy ${ }^{3}$ and Jennifer Y.F. Lau ${ }^{1,4}$

5

$6 \quad{ }^{1}$ Department of Experimental Psychology, University of Oxford, South Parks Road, Oxford, 7 OX1 3UD, UK

$8 \quad 2$ Department of Child and Adolescent Psychiatry, Psychosomatics and Psychotherapy, Ludwig-

9 Maximilian-University of Munich, Waltherstrasse 23, 80337 Munich, Germany

$10{ }^{3}$ Oxford Centre for Human Brain Activity, University of Oxford, Warneford Hospital, Oxford,

11 OX3 7JX, UK

$12{ }^{4}$ Department of Psychology, Institute of Psychiatry, Kings College London, 16 De Crespigny

13 Park, London, SE5 8AF, UK

15 Corresponding author:

16 Belinda Platt

17 Department of Child and Adolescent Psychiatry, Psychosomatics and Psychotherapy

18 Ludwig-Maximilian University of Munich

1923 Waltherstrasse

20 80337, Munich

21 Germany

22 Telephone: +49 $8954403981 \quad$ Fax: +498951605942

23 Email: belinda.platt@med.uni-muenchen.de 
24 Key words: adolescent depression, cognitive bias modification, visual-search task, dot-probe task

Abstract

Adolescence is a vulnerable time for the onset of depression. Recent evidence from adult studies suggests not only that negative attention biases are correlated with symptoms of depression, but that reducing negative attention biases through training can in turn reduce symptomology. The role and plasticity of attention biases in adolescent depression, however, remains unclear. This study examines the association between symptoms of depression and attention biases, and whether such biases are modifiable, in a community sample of adolescents. We report data from 105 adolescents aged 13-17 who completed a dot-probe measure of attention bias before and after a single session of visual search-based cognitive bias modification training. This is the first study to find a significant association between negative attention biases and increased symptoms of depression in a community sample of adolescents. Contrary to expectations, we were unable to manipulate attention biases using a previously successful cognitive bias modification task. There were no significant effects of the training on positive affect and only modest effects of the training, identified in post-hoc analyses, were observed on negative affect. Our data replicate those from the adult literature, which suggest that adolescent depression is a disorder associated

41 with negative attention biases, although we were unable to modify attention biases in our study.

42 We identify numerous parameters of our methodology which may explain these null training effects, and which could be addressed in future cognitive bias modification studies of adolescent 44 depression. 
47

48

Adolescence is a time of increased vulnerability for depression. A prospective cohort study

yielded one-year point prevalence estimates of episodes of major depressive disorder (MDD) that rose dramatically from around $2 \%$ in early adolescence (ages $13-15$ ), to $15 \%$ in middle adolescence (ages 15-18) (Hankin, et al., 1998). While for some adolescents these symptoms subside, for others they persist and can lead to long-term psychiatric problems (Knapp, McCrone, Fombonne, Beecham, \& Wostear, 2002; Weissman, et al., 1999). As emphasis grows on developing early treatments, more needs to be understood about how symptoms of depression arise and abate across this developmentally-sensitive juncture.

Prominent theories of adult depression have recently considered the association between heightened attention towards negative (versus neutral) stimuli and depression (De Raedt \& Koster, 2010; Ellenbogen, Schwartzman, Stewart, \& Walker, 2002; Koster, De Raedt, Goeleven, Franck, \& Crombez, 2005; Peckham, McHugh, \& Otto, 2010; Shane \& Peterson, 2007), with data suggesting that attention biases may play a causal role in the onset of depression (Browning, Blackwell, \& Holmes, 2013; Dandeneau \& Baldwin, 2004, 2009; Dandeneau, Baldwin, Baccus, Sakellaropoulo, \& Pruessner, 2007; MacLeod, 2012). In the present study, we address two research questions: (a) are symptoms of depression associated with increased attention towards negative stimuli in adolescents, as they are in adults? (b) are attention biases modifiable and associated with changes in negative affect in adolescents, as they are in adults?

\section{Attention biases and depressive conditions}

Reviews (De Raedt, et al., 2010; Peckham, et al., 2010) demonstrate overwhelming support for the presence of negative attention biases in currently depressed (Eizenman, et al., 2003; Gotlib, Kasch, et al., 2004; Gotlib, Krasnoperova, Yue, \& Joormann, 2004; Gupta \& Kar, 
2012; Joormann \& Gotlib, 2007; Leyman, De Raedt, Schacht, \& Koster, 2007; Rinck \& Becker, 2005; Suslow, Junghanns, \& Arolt, 2001) and dysphoric (Bradley, Mogg, Falla, \& Hamilton, 1998; Ellenbogen, et al., 2002; Koster, et al., 2005; Shane, et al., 2007) adults. Most studies have measured attention biases using the dot-probe task (MacLeod, Mathews, \& Tata, 1986), where participants are briefly exposed to a negative and a neutral stimulus presented simultaneously. A probe subsequently appears in the location of either the negative stimulus (congruent trial) or neutral stimulus (incongruent trial) and participants' reaction times (RT) to identify a characteristic of the probe (e.g. orientation) are measured. Negative attention biases are characterized by faster RTs to congruent trials and slower RTs to incongruent trials. Although some studies have failed to find evidence of negative attention biases in adults with depression (Karparova, Kersting, \& Suslow, 2005; MacLeod, et al., 1986; Mogg, Bradley, Williams, \& Mathews, 1993), this may be due to the conditions under which attention biases have been measured (De Raedt, et al., 2010; Peckham, et al., 2010), for example, stimuli may need to be self-relevant. There is also ongoing debate about whether stimuli need to be exposed for more than 1,000 milliseconds (ms) in order to observe depression-related biases (Peckham, et al., 2010).

Some studies have investigated whether negative attention biases also characterize adolescents with depression. An early study using the dot-probe task to compare clinically depressed adolescents and healthy controls found no group differences in attention bias, although it should be noted that the sample was relatively small ( $N=19$ depressed participants) (NeshatDoost, Moradi, Taghavi, Yule, \& Dalgleish, 2000). More recent dot-probe studies of larger samples suggest that depressed adolescents show attention biases towards sad (Hankin, Gibb, Abela, \& Flory, 2010) and angry (Salum, et al., 2013) (versus neutral) faces. Using an 
92 alternative task which measures attentional control, the Go/No-Go task, other studies have shown 93 speeded switching of attention to negative words (Maalouf \& Brent, 2012) and faces

94 (Ladouceur, et al., 2006) in depressed versus non-depressed adolescents. It is equally important 95 to examine the association between symptoms of depression and attention biases in non-clinical samples given overwhelming evidence that adolescent depression is a continuous (rather than discrete) disorder (Wesselhoeft, Sorensen, Heiervang, \& Bilenberg, 2013), with subthreshold depression in adolescence predicting depressive disorders and suicidal behavior in adulthood (Fergusson, Horwood, Ridder, \& Beautrais, 2005). Few studies have explored attention biases in dysphoric adolescents (De Voogd, Wiers, Prins, \& Salemink, 2014; Lonigan \& Vasey, 2009; Reid, Salmon, \& Lovibond, 2006), with one finding no correlation between attention bias and symptoms of depression (De Voogd, et al., 2014), and another finding a correlation that was better explained by anxiety symptoms (Reid, et al., 2006). A final study did not assess the relationship with symptoms of depression, but did observe a correlation between attention bias and negative affect (Lonigan, et al., 2009). Of note, none of these studies used the faces dotprobe task, as is typical in the adult literature and has been used to demonstrate attention biases in a study of clinically depressed adolescents (Hankin, et al., 2010). Modelling our hypotheses on findings in adults i.e. finding preferential attention engagement for negative faces, our first study hypothesis was that attention biases for negative faces (as measured using the dot-probe task) would characterize dysphoric adolescents.

\section{Can negative attention biases be modified such that they reduce negative affect?}

If the study of attention biases and adolescent symptoms of depression is to inform treatment models, a crucial question is whether these biases can be manipulated such that they initiate affect changes. Novel experimental paradigms, referred to as Cognitive Bias 
115 Modification (CBM), have been developed to manipulate cognitive patterns (including attention

116 biases; CBM-A) through repeated training. These paradigms could be useful for planning new

117 interventions if induced attention biases enable changes in affect or depressive symptomology.

118 The pioneering CBM-A paradigm is a modification of the dot-probe task (MacLeod, Rutherford,

119 Campbell, Ebsworthy, \& Holker, 2002), where the frequency of incongruent trials (trials where

120 the probe appears in the location of the neutral stimulus) is systematically increased throughout

121 the training session. This task reduced symptomology in students with mild-moderate symptoms

122 of depression (Wells \& Beevers, 2010) and adults with a previous diagnosis of depression

123 currently in remission (Browning, Holmes, Charles, Cowen, \& Harmer, 2012). A CBM-A task

124 based on a similar attention bias measure: Posner's cueing task (Posner, 1980), has provided

125 more mixed results, with one study suggesting effects are dependent on depression severity

126 (Baert, De Raedt, Schacht, \& Koster, 2010).

127 One criticism of these two tasks is that they do not expose participants to training stimuli

128 for long enough to facilitate attentional control (Kruijt, Putman, \& Van der Does, 2013). In

129 addition to an attention bias towards negative information, attentional control, the ability to shift

130 attention resources from one stimulus to another, may also be impaired in adult depression (De

131 Raedt, et al., 2010; Sanchez, Everaert, De Putter, Mueller, \& Koster, 2015). Poor attentional

132 control has been associated with increased symptoms of depression in children and adolescents

133 (Muris, Meesters, \& Rompelberg, 2007). A CBM-A paradigm based on the visual search task

134 (Hansen \& Hansen, 1988) may address this limitation. In this task participants are presented

135 with a matrix of 15 negative faces and a single positive face. Participants learn to disengage from

136 negative stimuli and selectively attend to the positive stimulus (identifying a smiling face as fast

137 as they can). Compared to a control training task, the paradigm is effective in reducing negative 
138 attention biases in adults with low self-esteem (Dandeneau, et al., 2004, 2009; Dandeneau, et al.,

139 2007), reducing stress (Dandeneau, et al., 2007), reducing the impact of a stress manipulation

140 (Dandeneau, et al., 2009), and increasing self-esteem (Dandeneau, et al., 2009; Dandeneau, et al., 141 2007).

142 Despite the relatively promising findings of previous CBM-A studies in relation to 143 depression, one should remain cautious about their clinical potential, not least because at least 144 one study failed to find positive effects on vulnerability for depression (Kruijt, et al., 2013). In 145 this study of dysphoric adults there was no evidence that the visual search CBM-A task modified 146 affect (Kruijt, et al., 2013), although the relatively small sample size $(\mathrm{N}=40)$ and the fact that the 147 task failed to modify attention bias in the first place, limits the interpretation of these null-effects. 148 Adolescence is a period of protracted brain maturation and possibly higher levels of 149 plasticity (Cohen Kadosh, Linden, \& Lau, 2013) - therefore we might predict that modifying 150 biases in this age range may be more effective than those data reported in previous adult studies. 151 On the other hand, immature pre-frontal networks in adolescence (Nelson, Leibenluft, McClure, 152 \& Pine, 2005) may reduce adolescents' ability to deploy top-down inhibitory control 153 mechanisms that are engaged in the CBM-A paradigm; therefore we may see weaker effects on 154 negative affect in this age group. The developmental-appropriateness of CBM-A paradigms has 155 been explored in relation to anxiety disorders. One review suggests that CBM-A paradigms may 156 be effective in reducing anxiety in children and adolescents (Lowther and Newman, 2014), 157 although a more recent review highlights the need to remain cautious about the clinical efficacy 158 of CBM-A for youth before more robust effects on symptomology are seen (Cristea, Mogoase, 159 David, \& Cuijpers, 2015). To date, just one study has investigated CBM-A in relation to 160 adolescent depression. De Voogd and colleagues administered two sessions of a visual search 
161 CBM-A training or a placebo-control training task to 32 adolescents aged 13-16 (De Voogd, et

162 al., 2014). Attention biases were measured before and after training using an assessment version

163 of the visual search training task described above, in which $50 \%$ of trials involved finding a

164 positive face in a matrix of negative faces, and 50\% of trials involved finding a negative face in a

165 matrix of positive faces. Attention bias was calculated by subtracting mean RT to negative

166 targets, from mean RT to positive targets. Although the CBM-A training paradigm appeared to

167 be effective in modifying attention biases, it remains unclear whether training effects transfer to

168 other attention bias measures e.g. the dot-probe task. Secondly, although no effect of CBM-A on

169 symptoms of depression was observed, negative affect may have been a better outcome for

170 detecting more subtle effects of CBM-A on depression. Our second aim was therefore to

171 examine the efficacy of the visual search task in dysphoric adolescents, but using the faces dot-

172 probe task to measure the effects on attention bias. Unlike De Voogd and colleagues, we also

173 investigated whether the training had an effect on positive and negative affect.

\section{Current aims and hypotheses}

175 In a single-session CBM-A training study, we addressed two outstanding questions in the

176 adolescent literature. First, we investigated whether negative attention biases (as measured by the

177 faces dot-probe task) were associated with symptoms of depression in a sample of adolescents.

178 Of note, we used a dot-probe stimulus duration of 500ms (a) in order to facilitate comparison of

179 the results with a previous study using the same paradigm and CBM-A procedure (Dandeneau, et

180 al., 2007) and (b) because we were concerned that a longer presentation time could not

181 distinguish between attention and elaborative biases. Second, we examined whether modifying

182 attention biases by increasing attentional control could alter negative affect in this sample. We

183 selected the visual search CBM-A task because of studies supporting its efficacy in modifying 
184 attention biases in adults (Dandeneau, et al., 2004, 2009; Dandeneau, et al., 2007) and

185 adolescents (De Voogd, et al., 2014) and because the task may enhance attentional control in

186 participants with symptoms of depression (Peckham, et al., 2010). Again, to facilitate

187 comparison with previous studies (Dandeneau, et al., 2007; De Voogd, et al., 2014), we used

188 negative and positive (rather than negative and neutral) training stimuli in the training task. We

189 also explored whether the effects of the attention training task were more pronounced in

190 participants with more symptoms of depression, who were more likely to show an initial

191 negative attention bias.

Materials \& Methods

193

194

195

196

197

198

199

200

201

202

203

204

205

206

\section{Participants and procedure}

A community sample of 112 adolescents aged 13-17 (mean age $=16$ years, 5 months; standard deviation $(\mathrm{sd})=0.76)$ were recruited through urban and rural secondary state schools and public advertisements in Oxfordshire, UK. The study session lasted 45 minutes. Adolescents recruited from schools were tested in groups at individual computers in a research laboratory at the University of Oxford. Adolescents recruited through public advertisements were tested individually in the same room and were given a $£ 10$ gift voucher for their participation. Participants first completed computerized measures of symptoms of depression, attention bias and affect. Participants were then randomly allocated to receive one of two CBM-A manipulations: learning to ignore emotionally negative (experimental group) or neutral (control group) stimuli. We oversampled participants in the experimental group ( $\mathrm{N}=75)$ compared to the control group $(\mathrm{N}=30)$ because of a priori expectations that there would be more variability in responsiveness to the experimental manipulation. Attention bias and affect ratings were measured again after training. Ethical approval for the study was provided by Oxford University 
207 Central University Research Ethics Committee (MSD/IDREC/C1/2010/56) and the study was

208 therefore performed in accordance with the ethical standards laid down in the 1964 Declaration 209 of Helsinki and its later amendments. Participants aged 16-17 provided written informed consent

210 and participants aged 13-15 provided written assent (written informed consent was provided by

211 their parents).

212 Measures

213 Symptoms of depression. Symptoms of depression were assessed in adolescents using 214 the Children's Depression Inventory (CDI) (Kovacs, 1992) and were available for 93\% (N=98)

215 of the final sample. In community samples the CDI correlates highly with other self-report

216 measures of low mood (Doerfler, Felner, Rowlison, Raley, \& Evans, 1988). Although the

217 original version contains 27 items, item 9 which assesses suicidal ideation was not administered

218 here because of ethical concerns about suggesting suicide to those who might not have thought

219 about it (Hanley \& Gibb, 2011; Nolen-Hoeksema, Girgus, \& Seligman, 1992; Smucker,

220 Craighead, Craighead, \& Green, 1986). In the current sample CDI scores ranged from 1 to 34

221 out of a possible 52 (mean=12.10,sd=6.6). Depression scores demonstrated high internal

222 consistency (Cronbach's $\alpha=0.85$ ). Since depression scores were non-normally distributed (K-S

223 test statistic $=0.14, \mathrm{df}=98, \mathrm{p}<0.001)$ a $\log _{10}(\mathrm{x}+1)$ transformation was applied and transformed

224 scores are reported hereafter.

225 Attention bias. Attention bias was assessed using a modified version of the dot-probe

226 task (Bradley, et al., 1998) (see Figure 1). Stimuli were five male and five female adult grey-

227 scale faces (participants were shown stimuli of their own gender) taken from the NimStim

228 dataset (Tottenham, et al., 2009). A negative (angry) and neutral expression of each face was

229 presented side-by-side. We chose to use angry (threatening) faces for the negative stimuli in line 
230 with much of the previous literature and because threatening faces are thought to elicit a

231 depressive reaction by depicting personal rejection whereas sad faces may depict the emotion of

232 an observer (Leyman, et al., 2007). Whether the negative face appeared on the left or the right

233 hand side was counterbalanced across trials. The face pairs were of resolution 505 dpi, measured

$234200 \times 257$ pixels, and were displayed on a black background. The face stimuli were presented for

$235500 \mathrm{~ms}$ and were immediately followed by a probe, which appeared in the location of one of the

236 preceding faces. The probe was two dots presented either vertically (' $\left.{ }^{\prime}\right)$ or horizontally ('..’) and

237 was displayed on the screen for $100 \mathrm{~ms}$. Participants were required to identify the probe

238 orientation using the ' $\mathrm{z}$ ' key for vertical orientation (':') and the ' $\mathrm{m}$ ' key for horizontal

239 orientation ('..'). The probe was presented for $100 \mathrm{~ms}$, rather than an unlimited or longer time

240 interval, to prevent elaborative stimulus processing and support the automatic attention process

241 we were trying to capture. Responses made within $1000 \mathrm{~ms}$ of probe onset were recorded. The

242 inter-trial interval varied randomly between $500 \mathrm{~ms}$ and $1250 \mathrm{~ms}$. Face-pairs and probes were

243 presented in a random order. There were two trial-types: congruent (the probe replaced the

244 negative face) and incongruent (the probe replaced the neutral face). In total there were 80 test

245 trials and 40 'filler' trials (where neutral expressions of each face were presented side by side).

246 Trials were presented in two blocks of 60 trials, preceded by 12 practice trials which gave

247 participants 'correct' or 'incorrect' feedback. The task was programmed in E-Prime.

248

249

250

251
[Insert Figure 1 here]

Affect ratings. Affect ratings were assessed using the Positive and Negative Affectivity Scale (PANAS) (Watson, Clark, \& Tellegen, 1988). The 20 -item scale provides both a positive and negative affect score. The scale has high reliability (Cronbach's $\alpha=0.83$ ) in adolescent 
252 samples (Garcia, Kerekes, Andersson, \& Archer, 2012). Affect ratings for both time points were

253 available for $98 \%(\mathrm{~N}=103)$ of the final sample.

254 Experimental and control training tasks

255 Both training tasks involved identifying a distinguishing feature in a 4 x 4 grid, over six 256 practice trials and a single block of 112 experimental trials (Dandeneau, et al., 2007) (see Figure

257 2). In both conditions, trials began with a fixation cross ('+') which appeared in the center of the 258 screen for $1000 \mathrm{~ms}$. This was followed by the $4 \times 4$ grid $(10,000 \mathrm{~ms})$. Participants were asked to 259 identify the target stimuli using the left button of the mouse. For the experimental training task, 260 the target stimulus was a positive (smiling) face, while the distracting stimuli were negative 261 (frowning) faces. For the control training task, the target stimulus was a five-petalled flower 262 while the distracting stimuli were seven-petalled flowers. Practice trials provided participants 263 with 'correct' or 'incorrect' feedback.

264 Stimuli in the experimental training condition were modified from the original task to 265 include 16 adolescent (instead of adult) faces selected from the NIMH Child Emotional Faces 266 Picture Set (NIMH-ChEFS)(Egger, et al., 2011). The faces were of resolution 300 dpi, measured $2678.5 \times 8.5 \mathrm{~mm}$ on the screen, and were presented in color on a grey background. Each of the 268 stimuli and presented for the same duration. The tasks were programmed in E-Prime.

Data preparation and statistical analysis

Attention bias was calculated by subtracting mean RT (ms) to congruent trials from incongruent trials, such that positive scores represented a negative attention bias (Dandeneau, et 
275 al., 2007; MacLeod, et al., 1986). Trials were excluded from analysis if the response was

276 inaccurate or if RT was less than 200ms or greater than two standard deviations from each

277 participant's mean RT (Ratcliff, 1993; Roy, et al., 2008). Of note, the mean error rate for dot-

278 probe trials was $16.60 \%(\mathrm{sd}=9.1)$. Dot-probe data from five participants (either pre- or post-

279 training task data) were incomplete due to a technical error during runtime and these subjects'

280 complete data were therefore removed. There was no reason to believe there was a systematic

281 pattern to the disruption and therefore the exclusion of these data is unlikely to have caused a

282 bias in the final sample. Two participants in the control training condition were excluded because

283 they obtained less than $65 \%$ accuracy on the task.

284 Data were analyzed using SPSS. In order to assess whether participants with more

285 symptoms of depression demonstrated a greater negative attention bias, a bivariate correlation

286 analysis between (transformed) symptoms of depression and baseline attention bias was

287 conducted. To test the main effect of the training manipulation (experimental, control) on

288 attention bias change scores (post-training-bias minus baseline-bias score) and the interaction

289 between training manipulation and (transformed) symptoms of depression (continuous variable),

290 a repeated-measures custom model ANOVA was used. Two similar custom model ANOVA tests

291 explored the effects of training manipulation and symptoms of depression on change scores for

292 negative affect and positive affect respectively. Differences in baseline demographic

293 characteristics and training performance between the experimental and control groups were

294 assessed using chi-square and t-tests.

295

\section{Results}

296

The final sample comprised 105 adolescents (Table 1).

297

[Insert Table 1 here] 
What is the relationship between symptoms of depression and attention bias?

299 A bivariate correlation (performed on data from the 98 (93\%) participants who provided depressive symptom scores) revealed that symptoms of depression correlated significantly with baseline attention bias (Pearson's $\mathrm{R}=0.20, \mathrm{p}=0.05$; Figure 3). Of note, symptoms of depression were also associated with negative $(\mathrm{N}=97$; Pearson's $\mathrm{R}=0.41, \mathrm{p}<0.001)$ and positive $(\mathrm{N}=97$; Pearson's $\mathrm{R}=-0.46, \mathrm{p}<0.001)$ affect. However, there was no evidence of a significant association between baseline attention bias and negative affect $(\mathrm{N}=104$; Pearson's $\mathrm{R}=0.09, \mathrm{p}=0.36)$, or between baseline attention bias and positive affect ( $\mathrm{N}=104$; Pearson's $\mathrm{R}=0.17, \mathrm{p}=0.09)$.

\section{Can attention biases be modified such that they improve negative affect?}

There were no significant differences between the two training conditions in terms of participants' age $\left(\mathrm{t}_{102}=0.70, \mathrm{p}=0.48\right)$, symptoms of depression $\left(\mathrm{t}_{96}=-1.53, \mathrm{p}=0.13\right)$, baseline attention bias $\left(\mathrm{t}_{103}=-0.95, \mathrm{p}=0.34\right)$, positive affect $\left(\mathrm{t}_{102}=0.10, \mathrm{p}=0.92\right)$, or negative affect $\left(\mathrm{t}_{45.45}=-\right.$ between the experimental training condition and the control condition $\left(\chi^{2}=1.09, p=0.30\right.$; Table 1). Task performance varied significantly between those who completed the experimental and those who completed the control training. Participants who performed the control training task were less accurate $\left(\mathrm{t}_{48.4}=3.70, \mathrm{p}<0.001\right.$, Cohen's $\left.\mathrm{d}=0.8\right)$ and took longer to correctly identify the target stimulus $\left(\mathrm{t}_{103}=-3.51, \mathrm{p}<0.001\right.$, Cohen's $\left.\mathrm{d}=0.8\right)$ than those who performed the experimental 317 training task. 
321 individuals received experimental or control training. Neither was there a main effect of

322 symptoms of depression $\left(\mathrm{F}_{1,94}=1.65, \mathrm{p}=0.20\right)$ or a significant two-way interaction between

323 condition and symptoms of depression $\left(\mathrm{F}_{1,94}=0.01, \mathrm{p}=0.93\right)$.

324 Change in affect. An ANOVA model (data on all relevant variables available for $\mathrm{N}=96$

325 participants) revealed no main effect of condition on change in negative affect $\left(\mathrm{F}_{1,92}=2.56\right.$,

$326 \mathrm{p}=0.11)$. There was also no evidence of a main effect of symptoms of depression $\left(\mathrm{F}_{1,92}=0.29\right.$,

$327 \mathrm{p}=0.59)$ on change in negative affect. A two-way interaction between condition and depressive

328 symptoms was marginally significant $\left(\mathrm{F}_{1,92}=3.93, \mathrm{p}=0.05\right.$, partial eta squared $\left.=0.04\right)$, suggesting

329 that the effect of the training task on change in negative affect varied as a function of

330 participants' symptoms of depression. In order to explore this interaction, a median-split variable

331 was created based on (transformed) depressive symptom scores (median=1.06) - and the effect

332 of training condition on change in negative affect was investigated using paired-samples t-tests

333 in each subsample separately. For participants with more symptoms of depression, those in the

334 experimental condition showed a significant reduction in negative affect following the training

335 (mean $=-0.44, \mathrm{sd}=1.1, \mathrm{t}_{30}=2.24, \mathrm{p}=0.03$, Cohen's $\mathrm{d}=0.3$ ), whereas depressed participants in the

336 control condition showed no significant change $\left(\mathrm{t}_{17}=-1.30, \mathrm{p}=0.21\right)$. Participants with fewer

337 symptoms of depression showed no significant change in negative affect in the experimental

338 condition $\left(\mathrm{t}_{34}=1.40, \mathrm{p}=0.17\right)$ or control condition $\left(\mathrm{t}_{11}=1.86, \mathrm{p}=0.09\right)$.

339 A similar ANOVA (data on all relevant variables available for $\mathrm{N}=96$ participants)

340 revealed no main effect on change in positive affect of condition $\left(\mathrm{F}_{1,92}=0.26, \mathrm{p}=0.62\right)$, no main

341 effect of symptoms of depression on change in positive affect $\left(\mathrm{F}_{1,92}=0.04, \mathrm{p}=0.85\right)$, and no

342 interaction between condition and symptoms of depression on change in positive affect

$343 \quad\left(\mathrm{~F}_{1,92}=0.29, \mathrm{p}=0.59\right)$. 


\section{Discussion}

Our findings demonstrate an association between symptoms of depression and negative attention bias in a community sample of adolescents. The attention bias training task used in the current study was unsuccessful in modifying attention bias or positive affect. Modest effects of the training on negative affect were observed for participants with more symptoms of depression, however these findings are interpreted with caution due to the post-hoc nature of analysis. This study is the first to demonstrate a positive association between negative attention biases and symptoms of depression in an unselected adolescent sample. This overall finding is in

352 line with previous studies of attention biases in clinically depressed (Eizenman, et al., 2003;

353 Gotlib, Kasch, et al., 2004; Gotlib, Krasnoperova, et al., 2004; Gupta, et al., 2012; Joormann, et 354 al., 2007; Leyman, et al., 2007; Rinck, et al., 2005; Suslow, et al., 2001) and dysphoric (Bradley, 355 et al., 1998; Ellenbogen, et al., 2002; Koster, et al., 2005; Shane, et al., 2007) adults.

356 Furthermore, it extends a study of adolescents which found an association between attention bias 357 and negative affect (Lonigan, et al., 2009) and supports an adolescent extension of cognitive 358 theories, in which depression is associated with attention (albeit weakly) as well as elaborative 359 (e.g. memory and interpretive) biases (Beck, 1967; Mathews \& MacLeod, 2005; Williams, 360 Watts, MacLeod, \& Mathews, 1997).

361 The size of the correlation between attention bias and depressive symptoms was 362 nevertheless relatively small (Pearson's $\mathrm{R}=0.2$ ), which may be related to the low reliability of the dot-probe task. Although the dot-probe has been established as the standard measure of attention bias, developing new measures which more reliably measure attention bias in youth depression is important for future research in this field. Studies of attention bias in adult depression have

366 recently used eye-tracking during passive viewing tasks. In a recent study of alcohol-related 
367 attention biases, eye-tracking was found to have superior internal reliability over traditional

368 behavioural reaction-time measures (Christiansen, Mansfield, Duckworth, Field, \& Jones,

369 2015). A meta-analysis of eye-tracking studies suggests the depressed versus non-depressed

370 adults show increased maintenance of attention towards dysphoric stimuli during passive-

371 viewing (extended presentation of multiple emotional stimuli without instruction) (Armstrong \&

372 Olatunji, 2012). The only study to date of youth depression found the opposite effects - that

373 youth with depression showed attentional avoidance (reduced maintenance) with sad stimuli

374 (Harrison \& Gibb, 2014). These findings indicate that eye-tracking may be a valuable tool for

375 investigating attention biases in youth depression, and that there is more to be understood about

376 the developmental aspects of attention biases in depression.

377 Future studies of restricted periods of childhood and adolescence could also help identify

378 developmental periods when associations between attention biases and symptoms of depression

379 first emerge. Our data suggest that by adolescence hypervigilance of negative cues is already

380 characteristic of depressive symptoms, but it is not clear when this linkage first emerges. It

381 should be noted here that in our version of the dot-probe task the probe was displayed for a

382 limited duration of $100 \mathrm{~ms}$. This duration is shorter than in many previous dot-probe studies and

383 may have yielded a somewhat elevated error rate (mean error rate $=16.60 \%, \mathrm{sd}=9.1$ ). However,

384 we also cannot exclude the possibility that the higher than expected error rate was due to our

385 sample being relatively young. Of note, a recent study by Britton and colleagues, also delivering

386 the dot-probe task to typically developing adolescents, found mean error rates (before the

387 exclusion of outliers) of 16-18\% (Britton, et al., 2013).

388 A critical clinical question is whether modifying attention biases has therapeutic potential

389 for adolescents. CBM-A approaches were initially a topic of great excitement, with early studies 
390

391

392

393

394

396

397

398

399

400

401

402

403

404

405

406

407

408

409

410

411

412

demonstrating significant effects of training on negative mood and vulnerability for adult depression (Baert, et al., 2010; Browning, et al., 2012; Dandeneau, et al., 2004, 2009;

Dandeneau, et al., 2007; MacLeod, 2012; Wells, et al., 2010). CBM-A paradigms have potential as an add-on to standard psychiatric treatment given the ease by which they can be administered and the fact that they complement existing treatment approaches by addressing more automatic information processing. Indeed, one of the first meta-analyses of CBM-A for anxiety suggested medium $(d=0.61)$ effect sizes, which may be even larger in clinical populations (Hakamata, et al., 2010) and are comparable with effect sizes of existing treatments for anxiety. More recent meta-analyses of CBM-A for anxiety and depression in adult (Cristea, Kok, \& Cuijpers, 2015; Heeren, Mogoase, Philippot, \& McNally, 2015) and youth (Cristea, Mogoase, et al., 2015; Lowther \& Newman, 2014) populations rightly call for caution in the recommendation of CBMA paradigms as clinical tools, since effects are not replicated in all studies, effect sizes are sometimes small, and studies which trial the effects of CBM-A paradigms are often poorly conducted or provide insufficient data to assess the methodological quality. Thus, the lack of positive effects of CBM-A on emotional vulnerability is not reason enough for rejecting the potential of CBM-A paradigms. Clarke and colleagues observed that of the 29 CBM-A studies which reported data on attention bias change (in addition to effects on emotional vulnerability), 26 showed that when attention bias was successfully changed, emotional vulnerability was modified too $(\mathrm{N}=16)$, and when attention bias was unsuccessfully modified, there was no effect on emotional vulnerability $(\mathrm{N}=10)$ (Clarke, Notebaert, \& MacLeod, 2014). Together these findings suggest that CBM-A paradigms do indeed have potential as clinical tools, but that more research is needed to understand why they are often ineffective in modifying attention biases in the first place. 
414 in youth (De Voogd, et al., 2014). Although this study is one of few to successfully modify attention bias without seeing an effect on emotional vulnerability, emotional vulnerability was

416 measured as depressive symptoms rather than positive or negative affect (which may be more 417 sensitive measures for detecting change). The CBM-A task was also designed to modify social 418 anxiety rather than depression.

419 In our study we chose to use the visual search task because of its previous success in 420 modifying attention biases and affect in adults and adolescents (Dandeneau, et al., 2004, 2009; 421 Dandeneau, et al., 2007; De Voogd, et al., 2014), and because we hypothesized that the training

422

423

424

could enhance general attentional control, which may in turn affect attentional engagement with negative stimuli specifically. However, we failed to find evidence that negative attention biases could be manipulated using a visual search attentional bias training task in our young sample.

This is the second published failure to replicate the effects of this training task on attention bias (Kruijt, et al., 2013). Nevertheless, before concluding that attention biases cannot be modified using this visual search paradigm in dysphoric adolescents, numerous alternative explanations are worth considering. One possibility is that attentional training effects simply do not transfer from one task to another i.e. whilst the dot-probe task measures attentional engagement with negative stimuli, the visual search CBM-A task trains engagement with positive stimuli and disengagement from negative stimuli. This may explain why De Voogd and colleagues found effects of the visual search CBM-A task on attention bias (De Voogd, et al., 2014) but we and others (Kruijt, et al., 2013) have not. Indeed, although we chose to use the dot-probe task to aid comparison with the previous literature, it has been shown to have relatively low reliability, particularly in non-clinical samples (Schmukle, 2005). A second possible explanation is that we 
436 trained attention to (coloured) adolescent faces, but measured attention bias to (grey-scale) adult

437 faces. We may therefore have trained an attention bias towards salient (coloured, age-adapted)

438 stimuli specifically, which did not transfer to less salient (grey-scale, adult) stimuli. The failure

439 to include a visual search measure of attention bias, and the lack of consistency in test stimuli,

440 are therefore limitations of the current study. A third possible explanation for our findings is that

441 training effects may only be expected in participants showing an attention bias at baseline. Using

442 the same ANOVA method reported for the full sample above, we explored post-hoc whether

443 participants with baseline attention bias (i.e. dot-probe bias score $>0$; $N=53$ ) benefited from

444 training. However, there remained no evidence of an effect of training group on any of the three

445 outcome measures (change in attention bias, negative mood, or positive mood), or of an

446 interaction between training group and symptoms of depression (all Ps $>0.2$ ). A fourth possible

447 explanation for the lack of effects is that multiple training sessions may be needed to elicit robust

448 effects on attention bias. The single-session nature of the CBM-A paradigm is another limitation

449 of the current study.

450 Some evidence to support the beneficial effect of the CBM-A task is supported by the

451 fact that the experimental training condition was associated with reduced negative affect (in

452 participants with higher levels of depressive symptoms). However, whilst it is possible that this

453 effect is mediated by a change in attention bias that was not detected by the dot-probe assessment

454 task, changes in affect may also have been due to non-specific differences (e.g. task difficulty)

455 between the two training conditions. Indeed, RT and accuracy data suggest that the experimental

456 (faces) task was significantly easier to perform than the control (flowers) task, replicating

457 findings from a recent study using the same CBM-A training tasks (Kruijt, et al., 2013).

458 Furthermore, when we added training task accuracy scores to our ANOVA model, thereby 
459

460

461

462

463

464

465

466

467

468

469

470

471

472

473

474

475

476

477

478

479

480

481

assessing whether it modified the effect of training on negative affect, the previously significant interaction between condition and symptoms of depression disappeared. Although it seems plausible that participants with more symptoms of depression were simply more rewarded from successfully completing the experimental task, since the condition $\mathrm{x}$ depression $\mathrm{x}$ training accuracy interaction was non-significant, we remain cautious in this interpretation due to the post-hoc nature of the analyses. Finally, it should be acknowledged that the sample sizes in the post-hoc t-test analyses were unequal due to oversampling in the experimental group. Smaller sample sizes in high depressed $(\mathrm{N}=18)$ and low depressed $(\mathrm{N}=12)$ participants allocated to the control condition could explain the lack of training effects in these groups.

Our study highlights the fragility of CBM-A data and the infancy of the attention bias literature in relation to adolescent depression. However, whilst remaining cautious about the clinical potential of CBM-A tasks for adolescent depression, the positive findings from CBM-A studies of adult depression, along with the finding that CBM-A paradigms generally modify emotional vulnerability when attention bias is successfully modified, suggests that exploration of the optimal paradigms and parameters needed for attention bias modification in adolescents is a worthy area of future research (Clarke, et al., 2014). There are numerous ways in which future CBM-A studies could be conducted in order to increase the chances of attention bias change. Firstly, CBM-A tasks may be more successful in altering biases if multiple training sessions are employed. Secondly, given our finding that symptoms of depression are associated with baseline attention bias, CBM-A studies of adolescents with more severe symptoms of depression may show stronger training effects. On the other hand, depression-related attentional control difficulties could mean that these participants show more difficulty in performing the CBM-A task. CBM-A studies of clinically depressed adolescents would nevertheless be of interest. 
482 Thirdly, Posner's cueing task has shown promise in the modification of attention biases in

483 depressed adults, albeit with positive effects on symptom severity emerging following a post-hoc 484 analysis (Baert, et al., 2010), as in the current study.

485

486

487

488

489

490

491

492

493

494

495

496

497

498

\section{Conclusion}

Adolescence is a period of vulnerability for depression, yet little is known about the role of negative information processing in the onset of depressive symptoms during this developmental period. Data from an unselected sample of adolescents suggest that, as has been demonstrated in adult studies, negative attention biases are associated with increased symptoms of depression. In contrast to previous studies, we found no evidence that the visual-search CBMA task could modify attention biases (as measured using the dot-probe task) or positive affect in our community sample of adolescents. Modest effects of the training on negative affect were observed for participants with more symptoms of depression, however these findings are interpreted with caution due to the post-hoc nature of analysis. Numerous complexities associated with measuring and modifying attention biases mean that further examination of these effects is needed before firm conclusions about the precise role of attention biases in adolescent depression, and their implications for clinical practice can be drawn. 


\section{Acknowledgements}

501

502 We thank the participants who gave their time to take part in this study and their parents and

503 teachers for facilitating their involvement. We are also grateful to Kevin Hilbert, Merel

504 Kerstholt, Marissa Waldemore and Sophie Raeder for their help in data collection.

505

506 


\section{References}

Armstrong, T., \& Olatunji, B. O. (2012). Eye tracking of attention in the affective disorders: a meta-analytic review and synthesis. Clinical Psychology Review, 32, 704-723. doi: 10.1016/j.cpr.2012.09.004

Baert, S., De Raedt, R., Schacht, R., \& Koster, E. H. (2010). Attentional bias training in depression: therapeutic effects depend on depression severity. Journal of Behavior Therapy and Experimental Psychiatry, 41, 265-274. doi: 10.1016/j.jbtep.2010.02.004

Beck, A. T. (1967). Depression: Clinical, experimental, and theoretical aspects. New York: Hoeber.

Bradley, B. P., Mogg, K., Falla, S. J., \& Hamilton, L. R. (1998). Attentional Bias for Threatening Facial Expressions in Anxiety: Manipulation of Stimulus Duration. Cognition and Emotion, 12, 737-753. doi: 10.1080/026999398379411

Britton, J. C., Bar-Haim, Y., Clementi, M. A., Sankin, L. S., Chen, G., Shechner, T., . . Pine, D. S. (2013). Training-associated changes and stability of attention bias in youth: Implications for Attention Bias Modification Treatment for pediatric anxiety. Developmental Cognitive Neuroscience, 4, 52-64. doi: 10.1016/j.den.2012.11.001

Browning, M., Blackwell, S. E., \& Holmes, E. A. (2013). The Use of Cognitive Bias Modification and Imagery in the Understanding and Treatment of Depression. Current Topics in Behavioral Neurosciences, 14, 243-260. doi: 10.1007/7854_2012_212

Browning, M., Holmes, E. A., Charles, M., Cowen, P. J., \& Harmer, C. J. (2012). Using attentional bias modification as a cognitive vaccine against depression. Biological Psychiatry, 72, 572-579. doi: 10.1016/j.biopsych.2012.04.014 
530 Christiansen, P., Mansfield, R., Duckworth, J., Field, M., \& Jones, A. (2015). Internal reliability

531 of the alcohol-related visual probe task is increased by utilising personalised stimuli and

532 eye-tracking. Drug and Alcohol Dependence. doi: 10.1016/j.drugalcdep.2015.07.672

533 Clarke, P. J., Notebaert, L., \& MacLeod, C. (2014). Absence of evidence or evidence of absence:

534 reflecting on therapeutic implementations of attentional bias modification. BMC

535 Psychiatry, 14, 8. doi: 10.1186/1471-244x-14-8

536 Cohen Kadosh, K., Linden, D. E., \& Lau, J. Y. (2013). Plasticity during childhood and

537 adolescence: innovative approaches to investigating neurocognitive development. $\mathrm{Dev}$

$538 \quad$ Sci, 16, 574-583. doi: 10.1111/desc.12054; 10.1111/desc.12054

539 Cristea, I. A., Kok, R. N., \& Cuijpers, P. (2015). Efficacy of cognitive bias modification

540 interventions in anxiety and depression: meta-analysis. British Journal of Psychiatry,

541 206, 7-16. doi: 10.1192/bjp.bp.114.146761

542 Cristea, I. A., Mogoase, C., David, D., \& Cuijpers, P. (2015). Practitioner Review: Cognitive

543 bias modification for mental health problems in children and adolescents: a meta-

$544 \quad$ analysis. Journal of Child Psychology and Psychiatry and Allied Disciplines. doi:

$545 \quad 10.1111 /$ jcpp. 12383

546 Dandeneau, S. D., \& Baldwin, M. W. (2004). The inhibition of socially rejecting information

547 among people with high versus low self-esteem: The role of attentional bias and the

548 effects of bias reduction training. Journal of Social and Clinical Psychology, 23, 584-

$549 \quad$ 602. doi: $10.1521 /$ jscp.23.4.584.40306

550 Dandeneau, S. D., \& Baldwin, M. W. (2009). The buffering effects of rejection-inhibiting

551 attentional training on social and performance threat among adult students.

552 Contemporary Educational Psychology, 34, 42-50. doi: 10.1016/j.cedpsych.2008.05.004 
553 Dandeneau, S. D., Baldwin, M. W., Baccus, J. R., Sakellaropoulo, M., \& Pruessner, J. C. (2007).

554 Cutting stress off at the pass: Reducing vigilance and responsiveness to social threat by

555 manipulating attention. Journal of Personality and Social Psychology, 93, 651-666. doi:

$556 \quad 10.1037 / 0022-3514.93 .4 .651$

557 De Raedt, R., \& Koster, E. (2010). Understanding vulnerability for depression from a cognitive

558 neuroscience perspective: A reappraisal of attentional factors and a new conceptual

559 framework. Cognitive, Affective, \& Behavioral Neuroscience, 1, 50-70. doi:

$560 \quad 10.3758 /$ cabn.10.1.50

561 De Voogd, E. L., Wiers, R. W., Prins, P. J., \& Salemink, E. (2014). Visual search attentional bias modification reduced social phobia in adolescents. Journal of Behavior Therapy and Experimental Psychiatry, 45, 252-259. doi: 10.1016/j.jbtep.2013.11.006

564

Doerfler, L. A., Felner, R. D., Rowlison, R. T., Raley, P. A., \& Evans, E. (1988). Depression in children and adolescents: a comparative analysis of the utility and construct validity of two assessment measures. Journal of Consulting and Clinical Psychology, 56, 769-772. doi:

Egger, H. L., Pine, D. S., Nelson, E., Leibenluft, E., Ernst, M., Towbin, K. E., \& Angold, A. (2011). The NIMH Child Emotional Faces Picture Set (NIMH-ChEFS): a new set of children's facial emotion stimuli. Int J Methods Psychiatr Res, 20, 145-156. doi: 10.1002/mpr.343

Eizenman, M., Yu, L. H., Grupp, L., Eizenman, E., Ellenbogen, M., Gemar, M., \& Levitan, R. D. (2003). A naturalistic visual scanning approach to assess selective attention in major depressive disorder. Psychiatry Research, 118, 117-128. doi: 10.1016/S01651781(03)00068-4 
576 Ellenbogen, M. A., Schwartzman, A. E., Stewart, J., \& Walker, C. D. (2002). Stress and selective

577

578

579

580

581

582

583

584

585

586

587

588

589

590

591

592

593

594

595

596

597

598

attention: the interplay of mood, cortisol levels, and emotional information processing. Psychophysiology, 39, 723-732. doi: 10.1017/s0048577202010739

Fergusson, D. M., Horwood, L. J., Ridder, E. M., \& Beautrais, A. L. (2005). Subthreshold depression in adolescence and mental health outcomes in adulthood. Archives of General Psychiatry, 62, 66-72. doi: 10.1001/archpsyc.62.1.66

Garcia, D., Kerekes, N., Andersson, A. C. A., \& Archer, T. (2012). Temperament, Character, and Adolescents' Depressive Symptoms: Focusing on Affect. Depression Research and Treatment, 2012, 8. doi: doi:10.1155/2012/925372

Gotlib, I. H., Kasch, K. L., Traill, S., Joormann, J., Arnow, B. A., \& Johnson, S. L. (2004). Coherence and specificity of information-processing biases in depression and social phobia. Journal of Abnormal Psychology, 113, 386-398. doi: 10.1037/0021843x.113.3.386

Gotlib, I. H., Krasnoperova, E., Yue, D. N., \& Joormann, J. (2004). Attentional biases for negative interpersonal stimuli in clinical depression. Journal of Abnormal Psychology, 113, 121-135. doi: 10.1037/0021-843x.113.1.121

Gupta, R., \& Kar, B. R. (2012). Attention and memory biases as stable abnormalities among currently depressed and currently remitted individuals with unipolar depression. Frontiers in Psychiatry, 3, 99. doi: 10.3389/fpsyt.2012.00099

Hakamata, Y., Lissek, S., Bar-Haim, Y., Britton, J. C., Fox, N. A., Leibenluft, E., . . Pine, D. S. (2010). Attention bias modification treatment: a meta-analysis toward the establishment of novel treatment for anxiety. Biological Psychiatry, 68, 982-990. doi:

10.1016/j.biopsych.2010.07.021 
599 Hankin, B. L., Abramson, L. Y., Moffitt, T. E., Silva, P. A., McGee, R., \& Angell, K. E. (1998). 600 Development of depression from preadolescence to young adulthood: Emerging gender 601 differences in a 10-year longitudinal study. Journal of Abnormal Psychology, 107, 128602 140. doi: $10.1037 / 0021-843 x .107 .1 .128$

603 Hankin, B. L., Gibb, B. E., Abela, J. R., \& Flory, K. (2010). Selective attention to affective 604 stimuli and clinical depression among youths: role of anxiety and specificity of emotion. 605 Journal of Abnormal Psychology, 119, 491-501. doi: 10.1037/a0019609

606

607

608

609

610

611

612

613

614

615

616

617

618

619

620
Hanley, A. J., \& Gibb, B. E. (2011). Verbal victimization and changes in hopelessness among elementary school children. Journal of Clinical Child and Adolescent Psychology, 40, 772-776. doi: 10.1080/15374416.2011.597086

Hansen, C. H., \& Hansen, R. D. (1988). Finding the face in the crowd: An anger superiority effect. Journal of Personality and Social Psychology, 54, 917-924. doi: 10.1037/00223514.54.6.917; id: 15510

Harrison, A. J., \& Gibb, B. E. (2014). Attentional Biases in Currently Depressed Children: An Eye-Tracking Study of Biases in Sustained Attention to Emotional Stimuli. Journal of Clinical Child and Adolescent Psychology, 1-7. doi: 10.1080/15374416.2014.930688

Heeren, A., Mogoase, C., Philippot, P., \& McNally, R. J. (2015). Attention bias modification for social anxiety: A systematic review and meta-analysis. Clinical Psychology Review, 40, 76-90. doi: 10.1016/j.cpr.2015.06.001

Joormann, J., \& Gotlib, I. H. (2007). Selective attention to emotional faces following recovery from depression. Journal of Abnormal Psychology, 116, 80-85. doi: 10.1037/0021$843 x .116 .1 .80$ 
621 Karparova, S. P., Kersting, A., \& Suslow, T. (2005). Disengagement of attention from facial

622

623

624

625

626

627

628

629

630

631

632

633

634

635

636

637

638

639

640

641 emotion in unipolar depression. Psychiatry and Clinical Neurosciences, 59, 723-729. doi: $10.1111 / \mathrm{j} .1440-1819.2005 .01443 . \mathrm{x}$

Knapp, M., McCrone, P., Fombonne, E., Beecham, J., \& Wostear, G. (2002). The Maudsley long-term follow-up of child and adolescent depression: 3. Impact of comorbid conduct disorder on service use and costs in adulthood. British Journal of Psychiatry, 180, 19-23. doi: 10.1192/bjp.180.1.19

Koster, E. H., De Raedt, R., Goeleven, E., Franck, E., \& Crombez, G. (2005). Mood-congruent attentional bias in dysphoria: maintained attention to and impaired disengagement from negative information. Emotion, 5, 446-455. doi: 10.1037/1528-3542.5.4.446

Kovacs, M. (1992). The Children's Depression Inventory (CDI) Manual. Toronto, Canada: Multi-Health Systems, Inc.

Kruijt, A. W., Putman, P., \& Van der Does, W. (2013). The effects of a visual search attentional bias modification paradigm on attentional bias in dysphoric individuals. Journal of Behavior Therapy and Experimental Psychiatry, 44, 248-254. doi: 10.1016/j.jbtep.2012.11.003

Ladouceur, C. D., Dahl, R. E., Williamson, D. E., Birmaher, B., Axelson, D. A., Ryan, N. D., \& Casey, B. J. (2006). Processing emotional facial expressions influences performance on a Go/NoGo task in pediatric anxiety and depression. Journal of Child Psychology and Psychiatry and Allied Disciplines, 47, 1107-1115. doi: 10.1111/j.14697610.2006.01640.x 
642 Leyman, L., De Raedt, R., Schacht, R., \& Koster, E. H. (2007). Attentional biases for angry 643 faces in unipolar depression. Psychological Medicine, 37, 393-402. doi:

$644 \quad 10.1017 / \mathrm{s} 003329170600910 \mathrm{x}$

645 Lonigan, C. J., \& Vasey, M. W. (2009). Negative affectivity, effortful control, and attention to 646 threat-relevant stimuli. Journal of Abnormal Child Psychology, 37, 387-399. doi: $647 \quad 10.1007 / \mathrm{s} 10802-008-9284-\mathrm{y}$

648 Lowther, H., \& Newman, E. (2014). Attention bias modification (ABM) as a treatment for child 649 650 and adolescent anxiety: a systematic review. Journal of Affective Disorders, 168, 125135. doi: $10.1016 / j . j a d .2014 .06 .051$

Maalouf, F. T., \& Brent, D. A. (2012). Child and adolescent depression intervention overview: what works, for whom and how well? Child and Adolescent Psychiatric Clinics of North America, 21, 299-312. doi: 10.1016/j.chc.2012.01.001

MacLeod, C. (2012). Cognitive bias modification procedures in the management of mental disorders. Current Opinion in Psychiatry, 25, 114-120. doi: 10.1097/YCO.0b013e32834fda4a

MacLeod, C., Mathews, A., \& Tata, P. (1986). Attentional bias in emotional disorders. Journal of Abnormal Psychology, 95, 15-20. doi: 10.1037/0021-843X.95.1.15

MacLeod, C., Rutherford, E., Campbell, L., Ebsworthy, G., \& Holker, L. (2002). Selective attention and emotional vulnerability: assessing the causal basis of their association through the experimental manipulation of attentional bias. Journal of Abnormal Psychology, 111, 107-123. doi: 10.1037/0021-843X.111.1.107 
663 Mathews, A., \& MacLeod, C. (2005). Cognitive vulnerability to emotional disorders. Annual 664 Review of Clinical Psychology, 1, 167-195. doi:

$665 \quad$ 10.1146/annurev.clinpsy.1.102803.143916

666 Mogg, K., Bradley, B. P., Williams, R., \& Mathews, A. (1993). Subliminal processing of 667 emotional information in anxiety and depression. Journal of Abnormal Psychology, 102, 668 304-311. doi: $10.1037 / 0021-843 X .102 .2 .304$

669 Muris, P., Meesters, C., \& Rompelberg, L. (2007). Attention control in middle childhood: 670 relations to psychopathological symptoms and threat perception distortions. Behaviour $671 \quad$ Research and Therapy, 45, 997-1010. doi: 10.1016/j.brat.2006.07.010

672 Nelson, E. E., Leibenluft, E., McClure, E. B., \& Pine, D. S. (2005). The social re-orientation of 673 adolescence: a neuroscience perspective on the process and its relation to 674 675 676 psychopathology. Psychological Medicine, 35, 163-174. doi: $10.1017 / \mathrm{S} 0033291704003915$

Neshat-Doost, H. T., Moradi, A. R., Taghavi, M. R., Yule, W., \& Dalgleish, T. (2000). Lack of Attentional Bias for Emotional Information in Clinically Depressed Children and Adolescents on the Dot Probe Task. Journal of Child Psychology and Psychiatry, 41, 363-368. doi: 10.1111/1469-7610.00620

Nolen-Hoeksema, S., Girgus, J. S., \& Seligman, M. E. (1992). Predictors and consequences of childhood depressive symptoms: a 5-year longitudinal study. Journal of Abnormal Psychology, 101, 405-422. doi: 10.1037/0021-843X.101.3.405

Peckham, A. D., McHugh, R. K., \& Otto, M. W. (2010). A meta-analysis of the magnitude of biased attention in depression. Depression and Anxiety, 27, 1135-1142. doi: 10.1002/da.20755; 10.1002/da.20755 
686 Posner, M. I. (1980). Orienting of attention. Quarterly Journal of Experimental Psychology, 32,

687

688

689

690

691

692

693

694

695

696

697

698

699

700

701

702

703

704

705

706

707

708 3-25. doi: 10.1080/00335558008248231

Ratcliff, R. (1993). Methods for dealing with reaction time outliers. Psychological Bulletin, 114, 510-532. doi: 10.1037/0033-2909.114.3.510

Reid, S., Salmon, K., \& Lovibond, P. (2006). Cognitive Biases in Childhood Anxiety, Depression, and Aggression: Are They Pervasive or Specific? Cognitive Therapy and Research, 30, 531-549. doi: 10.1007/s10608-006-9077-y

Rinck, M., \& Becker, E. S. (2005). A comparison of attentional biases and memory biases in women with social phobia and major depression. Journal of Abnormal Psychology, 114, 62-74. doi: 10.1037/0021-843x.114.1.62

Roy, A. K., Vasa, R. A., Bruck, M., Mogg, K., Bradley, B. P., Sweeney, M., ... Team, C. (2008). Attention bias toward threat in pediatric anxiety disorders. Journal of the American Academy of Child and Adolescent Psychiatry, 47, 1189-1196. doi: 10.1097/CHI.0b013e3181825ace

Salum, G. A., Mogg, K., Bradley, B. P., Gadelha, A., Pan, P., Tamanaha, A. C., . . Pine, D. S. (2013). Threat bias in attention orienting: evidence of specificity in a large communitybased study. Psychological Medicine, 43, 733-745. doi: 10.1017/s0033291712001651

Sanchez, A., Everaert, J., De Putter, L. M., Mueller, S. C., \& Koster, E. H. (2015). Life is ... great! Emotional attention during instructed and uninstructed ambiguity resolution in relation to depressive symptoms. Biological Psychology, 109, 67-72. doi: 10.1016/j.biopsycho.2015.04.007

Schmukle, S. C. (2005). Unreliability of the dot probe task. European Journal of Personality, 19, 595-605. doi: 10.1002/per.554 
709

710

711

712

713

714

715

716

717

718

719

720

721

722

723

724

725

726

727

728

729

730

731

Shane, M. S., \& Peterson, J. B. (2007). An evaluation of early and late stage attentional processing of positive and negative information in dysphoria. Cognition and Emotion, 21, 789-815. doi: 10.1080/02699930600843197

Smucker, M. R., Craighead, W. E., Craighead, L. W., \& Green, B. J. (1986). Normative and reliability data for the Children's Depression Inventory. Journal of Abnormal Child Psychology, 14, 25-39. doi: 10.1007/BF00917219

Suslow, T., Junghanns, K., \& Arolt, V. (2001). Detection of facial expressions of emotions in depression. Perceptual and Motor Skills, 92, 857-868. doi: 10.2466/pms.2001.92.3.857

Tottenham, N., Tanaka, J. W., Leon, A. C., McCarry, T., Nurse, M., Hare, T. A., ... Nelson, C. (2009). The NimStim set of facial expressions: judgments from untrained research participants. Psychiatry Research, 168, 242-249. doi: 10.1016/j.psychres.2008.05.006

Watson, D., Clark, L. A., \& Tellegen, A. (1988). Development and validation of brief measures of positive and negative affect: the PANAS scales. Journal of Personality and Social Psychology, 54, 1063-1070. doi: 10.1037/0022-3514.54.6.1063

Weissman, M. M., Wolk, S., Goldstein, R. B., Moreau, D., Adams, P., Greenwald, S., . . . Wickramaratne, P. (1999). Depressed adolescents grown up. Journal of the American Medical Association, 281, 1707-1713. doi: 10.1001/jama.281.18.1707

Wells, T. T., \& Beevers, C. G. (2010). Biased attention and dysphoria: Manipulating selective attention reduces subsequent depressive symptoms. Cognition and Emotion, 24, 719-728. doi: $10.1080 / 02699930802652388$

Wesselhoeft, R., Sorensen, M. J., Heiervang, E. R., \& Bilenberg, N. (2013). Subthreshold depression in children and adolescents - a systematic review. Journal of Affective Disorders, 151, 7-22. doi: 10.1016/j.jad.2013.06.010 
732 Williams, J. M. G., Watts, F. N., MacLeod, C., \& Mathews, A. (1997). Cognitive psychology and 733 emotional disorders (Vol. 2nd). Chichester, UK: Wiley.

734

735 


\section{Table $\mathbf{1}$ (on next page)}

Participant characteristics

a - Significant difference between those in the Experimental $(N=75)$ and Control $(N=30)$ conditions $(p<0.05)$ 


\begin{tabular}{llll}
\hline & Whole sample (105) & Experimental condition (N=75) & Control condition (N=30) \\
& Mean (sd) & Mean (sd) & Mean (sd) \\
\hline Age (N=104) & $16.39(0.8)$ & $16.43(0.8)$ & $16.31(0.8)$ \\
Number (and percentage) of females (N=104) & $92(87.6)$ & $67(89.3)$ & $25(83.3)$ \\
Depressive symptoms (N=98) & $12.10(6.6)$ & $11.26(5.8)$ & $14.00(7.8)$ \\
Baseline attention bias (ms) $(\mathrm{N}=105)$ & $0.31(26.5)$ & $-1.24(26.4)$ & $4.20(26.8)$ \\
Post-training attention bias (ms) $(\mathrm{N}=105)$ & $1.57(23.0)$ & $1.14(22.1)$ & $2.66(25.5)$ \\
Baseline positive affect (N=104) & $5.59(1.6)$ & $5.60(1.7)$ & $5.56(1.5)$ \\
Post-training positive affect $(\mathrm{N}=104)$ & $5.68(1.7)$ & $5.71(1.7)$ & $5.60(1.7)$ \\
Baseline negative affect $(\mathrm{N}=104)$ & $1.93(1.5)$ & $1.77(1.4)$ & $2.33(1.7)$ \\
Post-training negative affect $(\mathrm{N}=104)$ & $1.71(1.5)$ & $1.46(1.3)$ & $2.34(1.9)$ \\
CBM-A trial accuracy $(\%)(\mathrm{N}=105)^{\mathrm{a}}$ & $96.2(4.6)$ & $97.2(4.2)$ & $93.6(4.7)$ \\
CBM-A trial RT $(\mathrm{N}=105)^{\mathrm{a}}$ & $2848.1(615.9)$ & $2721.4(590.2)$ & $3165.0(571.2)$ \\
\hline
\end{tabular}

2 a - Significant difference between those in the Experimental $(\mathrm{N}=75)$ and Control $(\mathrm{N}=30)$ conditions $(\mathrm{p}<0.05)$ 
1

Dot-probe task parameters

The original stimuli, not displayed here due to copyright reasons, were grey-scale faces taken from the Nimstim dataset (Tottenham et al., 2009).

Congruent trial

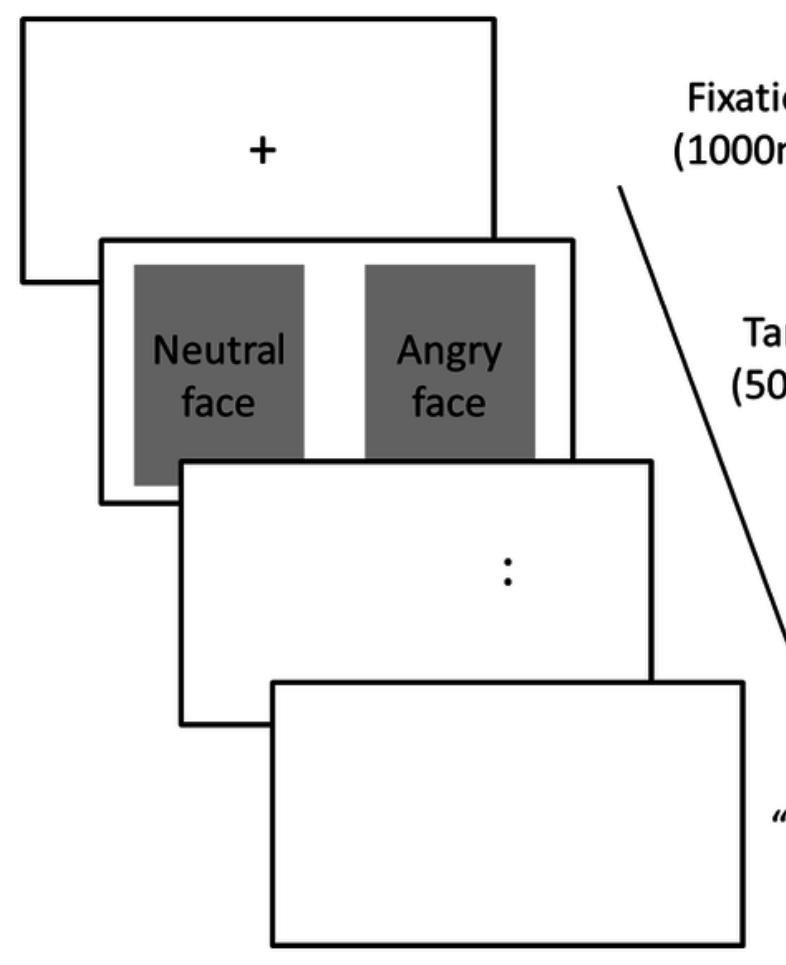

Incongruent trial

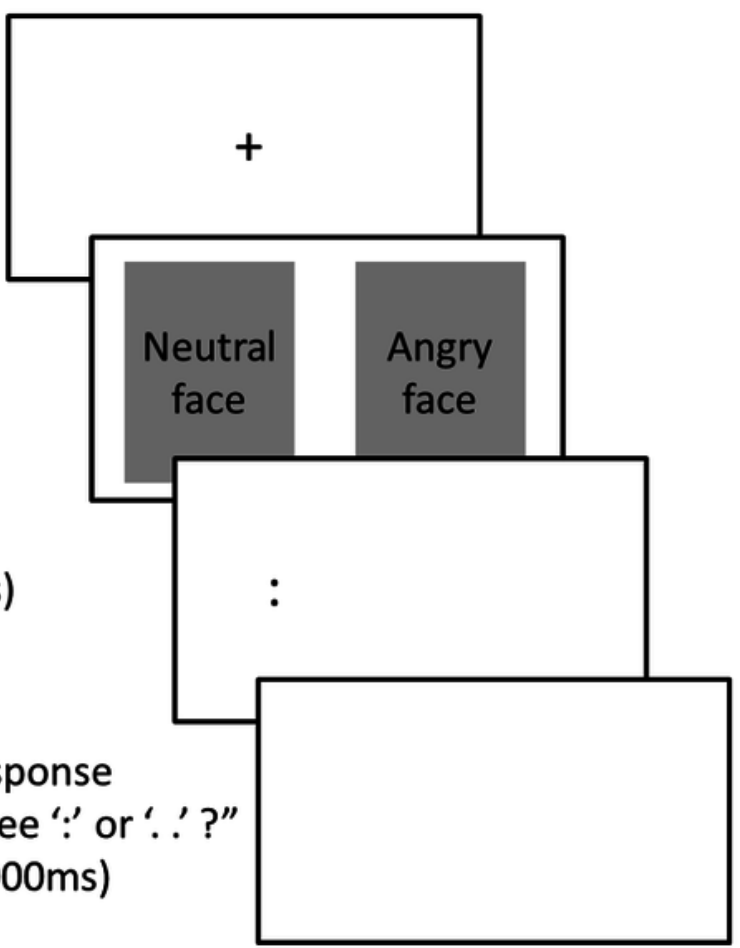


2

Experimental and control cognitive bias modification of attention (CBM-A) training tasks
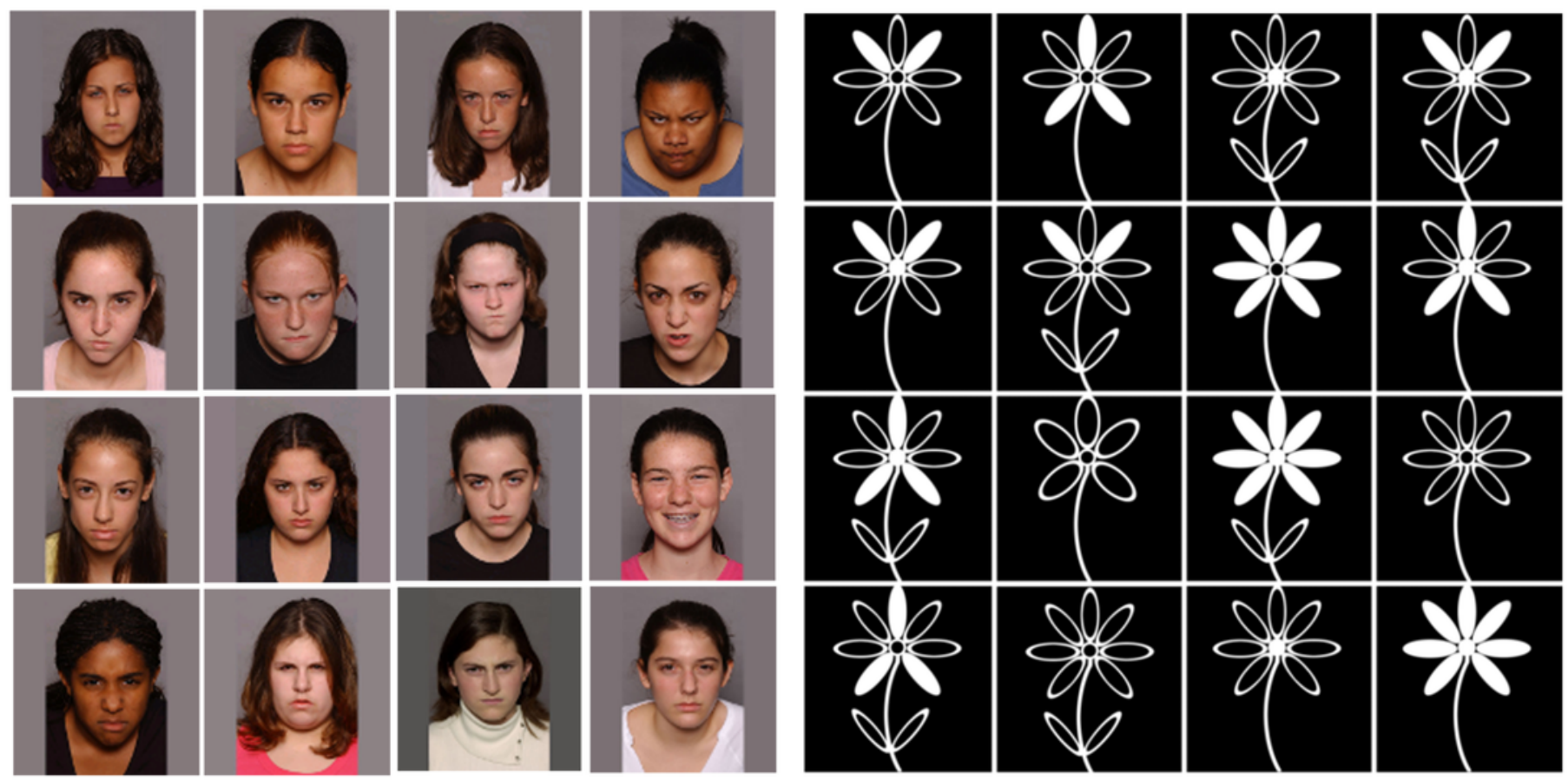
3

Association between baseline attention bias and depressive symptoms

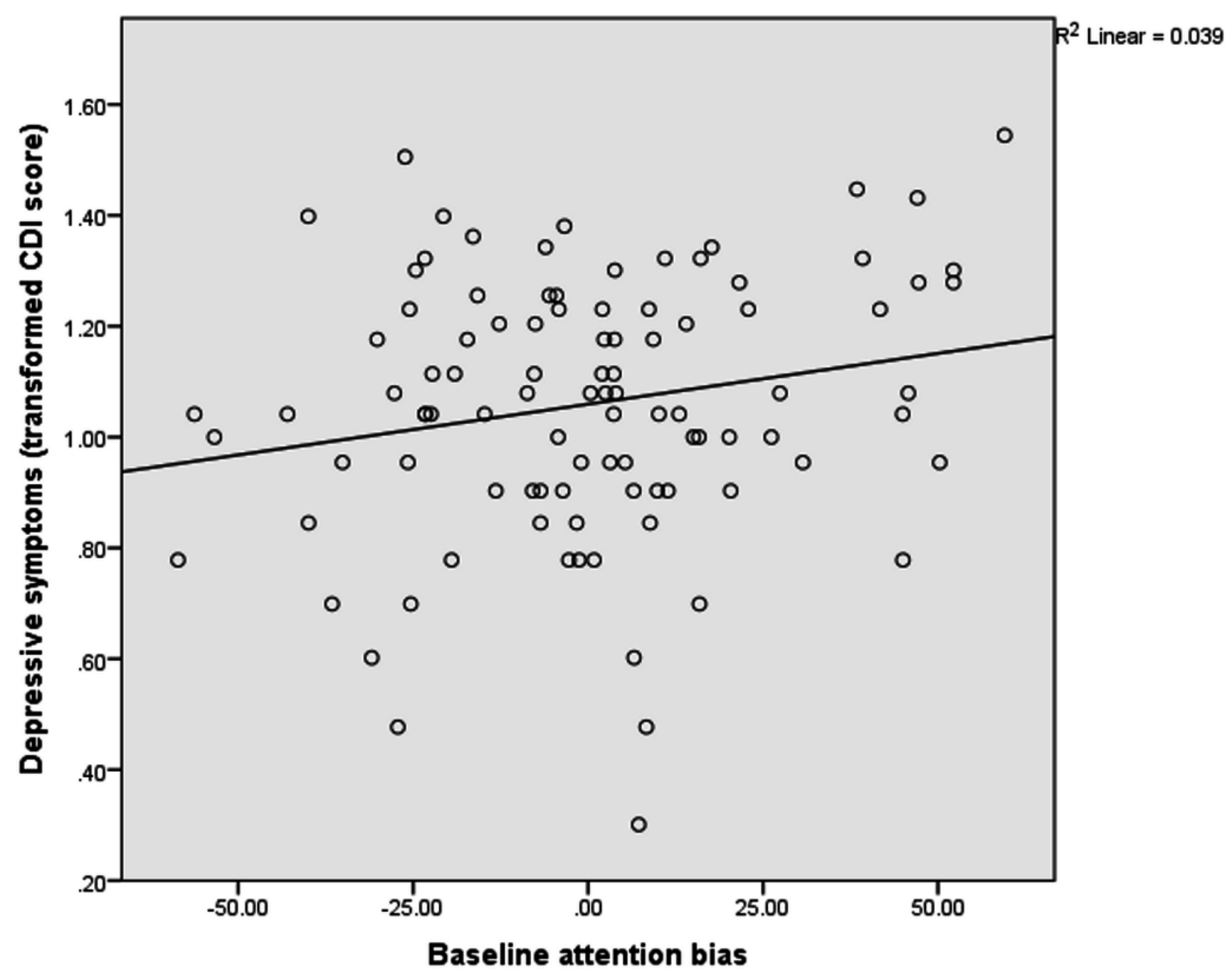

